\title{
Productive infection of HUVEC by HHV-8 is associated with changes compatible with angiogenic transformation
}

\section{Foglieni, ${ }^{1}$ S. Scabini, ${ }^{2}$ D. Belloni, ${ }^{2}$ F. Broccolo, ${ }^{1}$ P. Lusso, ${ }^{1,3}$ M.S. Malnati, ${ }^{15}$ E. Ferrero ${ }^{45}$} ${ }^{1}$ Unit of Human Virology, Department of Biological and Technological Research (DIBIT); ${ }^{2}$ nstitute of General Pathology, Medicine University of Milan; ${ }^{3}$ Department of Biomedical Science, University of Cagliari, Cagliari, Italy; ${ }^{4}$ Unit of Tumor Immunology, Department of Oncology, San Raffaele H Scientific Institute, Milan; ${ }^{5} \mathrm{MSM}$ and EF contributed equally to this work

(C2005, European Journal of Histochemistry

Kaposi's Sarcoma (KS) is an angioproliferative disease associated with human herpesvirus 8 (HHV-8) infection. We have characterized the morphologic and phenotypic modifications of HUVEC in a model of productive HHV-8 infection. HHV-8 replication was associated with ultra-structural changes, flattened soma and a loss of marginal folds and intercellular contacts, and morphologic features, spindle cell conversion and cordon-like structures formation. Phenotypic changes observed on cordon-like structures included partial loss and redistribution of CD31/PECAM-1 and VE-cadherin, uPAR upregulation and de novo expression of CD13/APN. Such changes demonstrate the induction, in HUVEC, of an angiogenic profile. Most of these findings are directly linked to HHV-8-encoded proteins expression, suggesting that HHV-8 itself may participate to the initial steps of the angiogenic transformation in KS.

Key words: Kaposi's Sarcoma, HUVEC, angiogenesis, herpesvirus.

Correspondence: Dr. Elisabetta Ferrero,

Unit of Tumor Immunology, Department of Oncology,

San Raffaele H Scientific Institute,

Via Olgettina 60, 20132 Milan, Italy

Tel: +39.2.2643.2612.

Fax:+39.2.2643.2611.

E-mail: ferrero.elisabetta@hsr.it

Paper accepted on June 20, 2005

European Journal of Histochemistry

2005; vol. 49 issue 3 (Jul-Sep): 273-284
$\mathrm{H}$ uman herpesvirus type 8 (HHV-8), also called Kaposi's sarcoma-associated herpesvirus, is an oncogenic virus belonging to the $\gamma$ 2-herpesvirus family, which is associated, in humans, with the development of unique malignancies (Boshof fand Wiess, 1998, Neipel and Fleckenstein, 1999; Cesarman et al., 1995; Soulier et al., 1995). Among them, Kaposi's sarcoma (KS), an angioproliferative disease occuring in four different clinical-epidemiological forms, has been the first to be directly associated with HHV-8 infection (Chang et al., 1994).

Indeed, the finding of a high HHV-8-DNA load in tumor tissues derived from all clinical forms of $\mathrm{KS}$ together with the restricted intra-lesion expression of HHV-8 latency-associated nuclear antigen (LANA 1) (Dupin et al., 1999; Boshoff et al., 1995; Sturzl et al., 1997, 1999; Staskus et al., 1997), which increases with the clinical stage of $\mathrm{KS}$, strongly argue for a direct involvement of HHV-8-encoded proteins in tumor establishment and progression (Malnati et al., 2003; Ensoli et al., 2001).

$\mathrm{KS}$ is a peculiar form of vascular neoplasm characterized by infiltration of lymphomononuclear cells, presence of activated proliferating endothelial cells forming abnormal blood vessels (slit-like vessels), extravasation of red blood cells and proliferation of spindle-shaped cells expressing endothelial and macrophage markers that are considered the bona fide tumoral cellular elements of KS (Boshoff et al., 1995; Fiorelli et al., 1998; Nickoloff et al., 1989; Uccini et al., 1994, 1997).

The establishment of a reproducible in vitro model for investigating the effects of HHV-8 infection and propagation on endothelial cells may be crucial to define the hierarchy of pathogenic events that lead to progressive angiogenic transformation and tumor establishment. Several reports have documented that HHV-8 can infect primary endothelial cells such as human bone marrow microvascu- 
lar endothelial cells, primary human umbilical vein endothelial cells (HUVEC) and human dermal microvascular endothelial cells (DMVEC) (Flore et al., 1998; Ciufo et al., 2001).

All these types of endothelial cells were latently infected by HHV-8, as assessed by viral proteins expression (such as LANA-1), acquired a spindloid morphology showing a prolonged survival time (few months) in culture. In one case, cell lines expressing telomerase activity and anchorage-independent growth have been obtained; however, in this model, only a small fraction of cells ( $1-3 \%$ ) expressed LANA-1 antigen (Flore et al., 1998).

In spite of these results, none of the experimental models hitherto described efficiently supports productive HHV-8 infection; therefore, such models are not suitable to investigate the potential initiating and promoting effects of viral lytic proteins. Indeed, several genes expressed during the viral lytic cycle encode homolog of cellular proteins involved in signal transduction, apoptosis and cellular regulatory pathways (Dagna et al., 2003; Dourmishev et al., 2003); all these viral gene products are good candidates for playing a pathogenetic role in KS tumor formation through autocrine and paracrine mechanisms (Ensoli et al., 1989, 2001). Recently, productive infection of primary human endothelial cells has been obtained in vitro using a recombinant virus engineered to monitor HHV-8 replication (Gao et al., 2003). In this system, however, the endothelial cell modifications that led to homogeneous, long-surviving cultures of $\mathrm{HHV}$-8 latently infected spindleshaped cells have not been fully investigated ( $G$ ao et al., 2003). To elucidate the oncogenic potential of $\mathrm{HHV}-8$, we have developed a model of infection of primary human endothelial cells (HUVEC) grown in absence of heparin and endothelial mitogens such as VEGF and $\beta$-FGF using HHV-8-containing supernatants derived from the EBV-negative human lymphoma B-cell line BCBL-1, particularly enriched in cellular growth factors and cytokines (Mercader et al. 2000). Herein, we describe the morphologic and phenotypic changes occurring in HUVEC cells in concomitance with HHV-8 infection.

These findings altogether support the hypothesis of a direct involvement of HHV-8 in the initiation and promotion of an angiogenic transformation of endothelial cells.

\section{Materials and Methods}

\section{HHV-8 viral stock}

HHV-8 viral stocks were prepared from the culture supernatant of unstimulated BCBL-1 cells that had been maintained in culture for less than 3 months in the absence of chemical stimuli. BCBL1 cells were grown in RPMI 1640 supplemented with 10\% heat-inactivated fetal bovine serum (FBS) (PAA Labour, Linz, Austria)), $100 \mathrm{IU} / \mathrm{mL}$ penicillin, $100 \mu \mathrm{g} / \mathrm{mL}$ streptomycin and $5 \times 10^{-5} \mathrm{M}$ 2-mercaptoethanol. Virus-containing cell culture supernatant was spun at $1400 \times \mathrm{xg}\left(15 \mathrm{~min}, 4^{\circ} \mathrm{C}\right)$ and filtered through a $0.4-\mu \mathrm{m}$ filter (Millipore) to exclude residuals cells and debris. Precleared BCBL-1 supernatant was aliquoted and frozen at $-80^{\circ} \mathrm{C}$ until used for infection.

\section{HUVEC infection}

Endothelial cells were obtained by perfusing with collagenase human umbilical veins from cords, provided by the Ostetricia and Ginecologia Department of the San Raffaele Hospital. The cells were grown in plastic flasks until they reached confluence in medium 199 (BioWhittaker, Verviers, Belgium) containing $20 \%$ heat-inactivated FBS and $1 \%$ retinal derived factor as described (Ferrero et al., 1995).

Primary endothelial cells were cultured without heparin that decreases the binding of H HV-8 to the target cells, and without endothelial growth factors (e.g. VEGF, $\beta-F G F$ ), that drive endothelial-cell differentiation. Moreover, the cells were used within the second passage, to avoid the loss of specific receptors (e.g. TNFR2), which typically occurs after prolonged culture periods.

BCBL-1 culture supernatant, containing between $5 \times 10^{5}$ and $2 \times 10^{6} \mathrm{HHV}-8$ genome equivalents/mL, was added to sub-confluent HUVEC cultures $\left(1 \times 10^{4}\right.$ cells in transwell chambers; $5 \times 10^{4}$ cells in $16 \mathrm{~mm}$ squared cover slips; $1 \times 10^{5}$ cells in 24 well plates).

After overnight incubation $(0 / N)$, the supernatant was discarded, cell cultures were washed once to remove dead cells and complete fresh medium was re-added to the cultures. Complete medium was replaced every two days and controls and infected cells were carried on under equivalent culture conditions.

For time course experiments the first sample considered (day 0 ) was collected 2 hours after an 
extensive wash-out of the $0 / \mathrm{N}$ infected cultures.

At fixed times all the culture supernatant was replaced and HHV-8-DNA accumulation evaluated on the discarded supernatant. In the same experiments control HUVEC cultures were challenged with aliquots of BCBL-1-derived supernatant subjected to three rounds of inactivation by UV light (16 J/m²; Lusso et al., 1991).

\section{Real-time PCR assay and sample preparation}

Quantification of HHV-8 DNA was performed using the TaqMan technology on an ABI PRISM 7700 SDS (Applied Biosystems, Foster City, CA). Briefly, primers and probe for the quantitative assay were derived from the KS330 Bam233 fragment of the conserved region ORF26 using the Primer Express software (Applied Biosystems, Foster City, CA). PCR was performed by activation of the uracyl-N-glycosylase (UNG) $\left(50^{\circ} \mathrm{C}\right.$ for $\left.2 \mathrm{~min}\right)$, followed by the AmpliTaq Gold activation $\left(95^{\circ} \mathrm{C}\right.$ for $\left.15 \mathrm{~min}\right)$ and by 40 cycles of amplification (denaturation step $15 \mathrm{~s}$ at $95^{\circ} \mathrm{C}$; annealing /extension step $1 \mathrm{~min}$ at $60^{\circ} \mathrm{C}$ ).

Primers and probes were used at the final concentration of 0.3 and $0.2 \mathrm{M}$ respectively. Known amounts of a plasmid carrying the ORF 26 fragment amplified by the same primers were used to obtain a reference curve for quantification of the HHV-8 load.

Supernatant (1 mL) from HHV-8-infected cultures was centrifuged $\left(1,500 \mathrm{~g}, 15 \mathrm{~min}, 4^{\circ} \mathrm{C}\right)$ and cell debris removed. Pre-cleared samples were subjected to high-speed centrifugation $(26,000$ $\mathrm{xg}, 90 \mathrm{~min}, 4^{\circ} \mathrm{C}$ ) to concentrate viral particles.

A synthetic calibrator DNA ( $10^{4}$ copies of calibrator/sample) was added prior to DNA extraction and co-amplified with the HHV-8 target sequence by real-time PCR. Primers and calibrator probe were specifically selected in order to avoid cross-hybridization with the HHV-8 sequences. DNA extraction from spun material was performed using a phenol-chloroform method, as described (Locatelli et al., 2000), and purified material was resuspended in a final volume of $100 \mu \mathrm{L}$ of $A E$ buffer. Ten microliters of the purified material were tested in each $P C R$ reaction in triplicate to measure both the calibrator and the HHV-8 copy number.

Data were normalized based on the recovery rate of the calibrator DNA as described (Broccolo et al., 2002).

\section{Transmission Electron Microscopy}

$5 \times 10^{4}$ HUVEC were plated on transwell polycarbonate filters and allowed to reach confluence 5 days before the infection. Infection was carried on as above described, and samples were overnight fixed at $4^{\circ} \mathrm{C}$ with $2.5 \%$ glutaraldehyde in $0.1 \mathrm{M}$ cacodylate buffer, $\mathrm{pH}=7.4$, and washed in cacodylate buffer. After that, the filters were excised and processed for inclusion in EmBed-812 resin as described (Ferrero et al., 1998).

Thin sections on copper grids were stained with uranyle acetate and lead citrate and were analysed at transmission electron microscope (Jeol 100 CX, Jeol Tokio, Jp) equipped with a digital camera system. All the reagents and supplies for TEM were from Electron Microscopy Sciences (Fort Washington, PA, USA).

\section{Cytofluorimetric analysis}

HUVEC were collected by brief exposure to $0.25 \%$ trypsin/1 mM EDTA (Invitrogen Corp., Carlsbad, CA, USA) and washed twice in ice-cold phosphate buffered $0.9 \%$ saline containing $1 \%$ FBS (FACS buffer). Cells were suspended in a final volume of $100 \mu \mathrm{L}$ of FACS buffer, incubated with anti uPAR mAb (R2, $2 \mu \mathrm{g} / \mathrm{mL})$, kindly provided by $\mathrm{E}$ Rønne and G Høyer-Hansen (Finsen Laboratory Copenaghen, Denmark), and then washed twice with FACS buffer and incubated with a FITC-conjugated goat-antimouse IgG polyclonal antibody (Zymed, San Francisco, CA, USA) for 30 min. All these steps were performed on ice. After washing, fluorescence was measured with a FACScan (Becton Dickinson, Mountain View, CA, USA) and the results were analyzed using the CellQuest software.

\section{Immunohistochemistry, immunofluorescence and confocal microscopy}

HUVEC plated and infected on $15 \mathrm{~mm}$ square glass coverslips were fixed in $2 \%$ paraformaldehyde in Dulbecco's PBS (DPBS), washed in DPBS then submitted to double indirect immunofluorescence combined with immunohistochemistry (IHC) staining. Combinations of monoclonal and polyclonal antibodies (Abs) against HHV-8 LANA-1 (MAb antiORF 73, ABI, Columbia, Maryland, USA) and V-IL6 (PAb anti ORF K2, ABI Columbia, Maryland, USA), human CD31 (MAb clone M89D3: Ferrero et al., 1995), human VE-cadherin ( MAb 
BV9, VE-cad, kindly provided by Monica Corada, IFOM, Milan Italy), Ki-67 (MAb clone MIB-1 from Immunotech, Hamburg, Germany), human CD13/aminopeptidase N (MAb CD13/APN, clone WM15 from PharMingen, San Diego, CA, USA), and Phalloidin-TRITC (Sigma Immunochemicals, St Louis MO, USA) were employed. Both antibodies against viral proteins were trated on BCBL-1 cultures to assess working dilutions and pattern of localization. The cells were first incubated with a blocking solution ( $1 \%$ BSA in DPBS, 30 minutes, $\mathrm{RT})$, then with the primary $A b$ diluted in blocking solution ( 2 hours, RT), and finally, after 30 minutes washing in DPB with a fluorochromeconjugated secondary $\mathrm{Ab}$ (rabbit-anti-mouse IgFITC, DAKO, Denmark, goat-anti-rabbitAlexaFluor 488, Molecular Probes, Eugene, OR, USA) in DPBS ( 30 minutes RT).

Double staining was obtained by superimposing the same procedure with a different primary $\mathrm{Ab}$ and a differently labeled secondary Ab (rabbit-anti-mouse Ig-TRITC, DAKO), or by applying Phalloidin-TRITC. LANA-1 was revealed by IHC with the $A B C$ method ( $A B C$-élite kit, Vector, Burlingame, $C A$, USA), prior to the other staining, in order to improve the labelling intensity.

For each experiment uninfected HUVEC and cultures where non immune sera substituted primary antibodies served as negative controls. Before adding Phallodin or anti-0RF73 or antiORFK2 mAbs, HUVEC were permeabilized with Triton-X100 in DPBS $(0,1 \%, 10$ minutes RT). When indicated, DAPI (Sigma Immunochemicals, St Louis MO, USA) nuclear staining $(0,2 \mathrm{nM}$, 20 minutes RT) was performed following the immunostaining.

The slides, abundantly washed in DPBS and mounted with Fluorsave (Calbiochem, Merck Eurolab Srl., Milan, Italy), were analyzed with the support of a confocal microscope Leica TCS SP2 (Leica Microsystems, Heidelberg, GmbH). 3D maximum projections were obtained from single channel-collected Z-series of images, and were superposed by Adobe Photoshop software. IHC was evaluated at conventional light microscope (BH2, Olympus, Melville, NY, USA) and digital picture were taken with the help of an Olympus C4040 zoom digital camera. Each experiment was repeated at least three times.

\section{Results}

\section{Infection of HUVEC with HHV-8}

HUVEC monolayers were exposed to the HHV-8containing culture supernatant of unstimulated BCBL-1 cells and productive infection was monitored by measuring the accumulation over time of HHV-8 DNA in the culture supernatants (Figure 1a), as well as the expression at different time points of two HHV-8 encoded proteins, LANA-1 (ORF 73) and v-IL-6 (ORF K2) (Figure $1 \mathrm{c}-\mathrm{e}$ ). We choose this experimental approach avoiding confounding effects on HUVEC due to the residual presence of well-known pro-differentiative agents such as TPA and PMA. The viral DNA load was measured using a novel real-time PCR approach that, owing to a synthetic DNA calibrator added before any sample manipulation, allows controlling both for the efficiency of the extraction procedure and for the presence of Taq-polymerase inhibitors, thus avoiding miscalculation of the viral load. The peak of viral DNA accumulation, a 100-fold increase over the residual DNA load measured after extensive culture washing, (time 0 , see Materials and Methods) was reached 36-48 hours post-infection (Figure 1a). In comparison, the peak of UV inactivated HHV-8 mock decreased quickly (Figure 1a).

To further document the productive infection of HUVEC, the expression of LANA- 1 and VIL- 6 viral proteins was examined by light and confocal microscopy at different time-points. Starting from 24 hours after infection (tI), v-IL-6 displayed a granular cytoplasmic pattern in few slightly altered cells (Figure 1c), while, at day (t7), it showed a peripheral distribution (Figure $6 \mathrm{c}, \mathrm{d}$ ). Anti-LANA1 labeled almost exclusively the nuclei of elongated cells from day 3 (t3) (Figure $1 d, e$ ) to $t 7$, which corresponded to $20-30 \%$ of the cells while normally shaped cells were negative (not shown). Similarly to $\mathrm{v}$-IL6, the expression intensity of LANA-1 (Figure $5 b$ ) weakened over time. No evidence of coexpression of LANA-1 and VIL-6 was observed at any time point.

Further evidence of productive HHV-8 infection in HUVEC cells was obtained by TEM performed at the peak (48h) of the infection as determined by viral DNA accumulation. In each thin section about $10-20 \%$ of the cells contained virus-like structures both in the nucleus and in the cytoplasm (Figure $1 \mathrm{~b}$, inserts). The infected cells were thin and elongated, 
similar to the spindle cells that characterize KS lesions. The nuclei of infected cells displayed fine chromatin with small chromocenters, the cytoplasm was swollen, without a clearly organized Golgi, rich in rough reticulum vescicles but poor in lysosomes, and with round mitochondria (Bosman et al., 1996). These cells failed to show the marginal folds, typically protruding from the surface of normal endothelial cells, and their cellular contacts were weakened or lost (Figure 1b, insert). Interestingly, multivesicular and hyaline bodies, observed by some authors in KS lesions, were undetectable, as were Weibel-Palade bodies, the regulated secretory organelles of endothelial cells (Michaux et al., 2004).

Altogether, these findings clearly indicated that HUVEC monolayers infected by a HHV-8 supernatant derived from unstimulated BCBL-1 cells were able to sustain both latent and lytic HHV-8 infection.

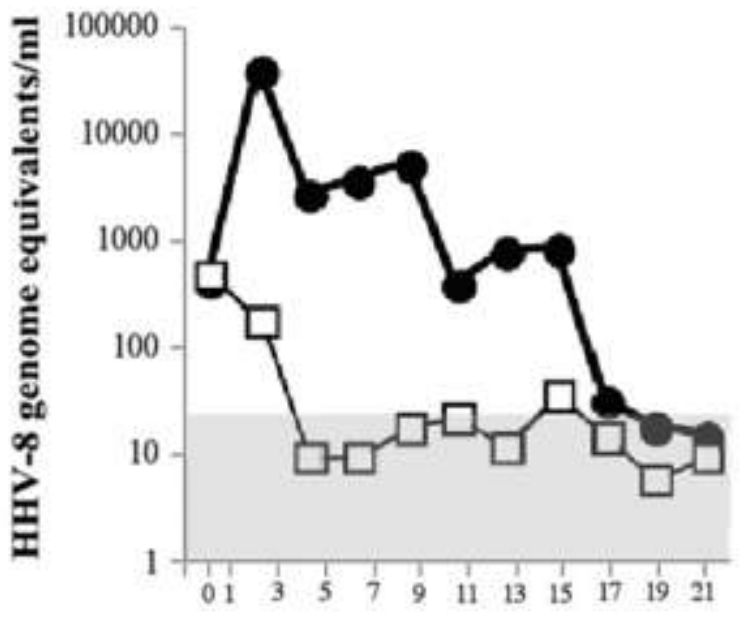

DAYS

a

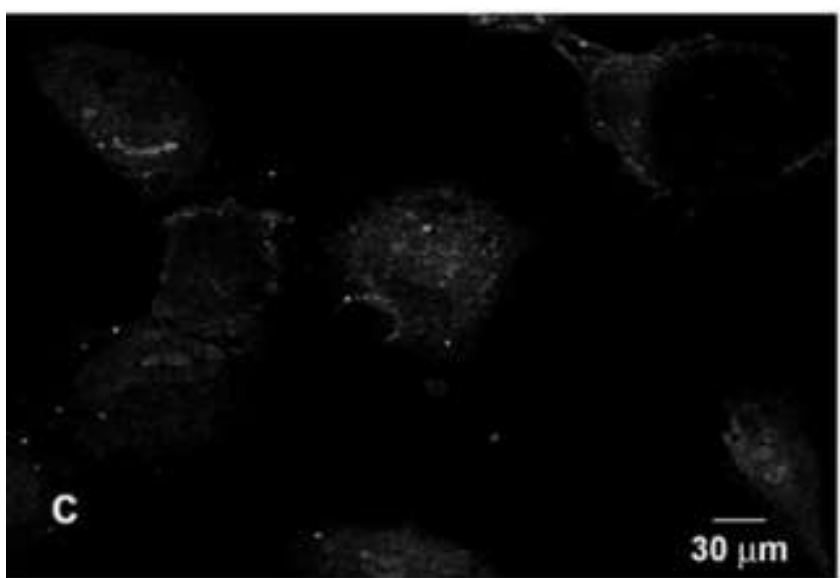

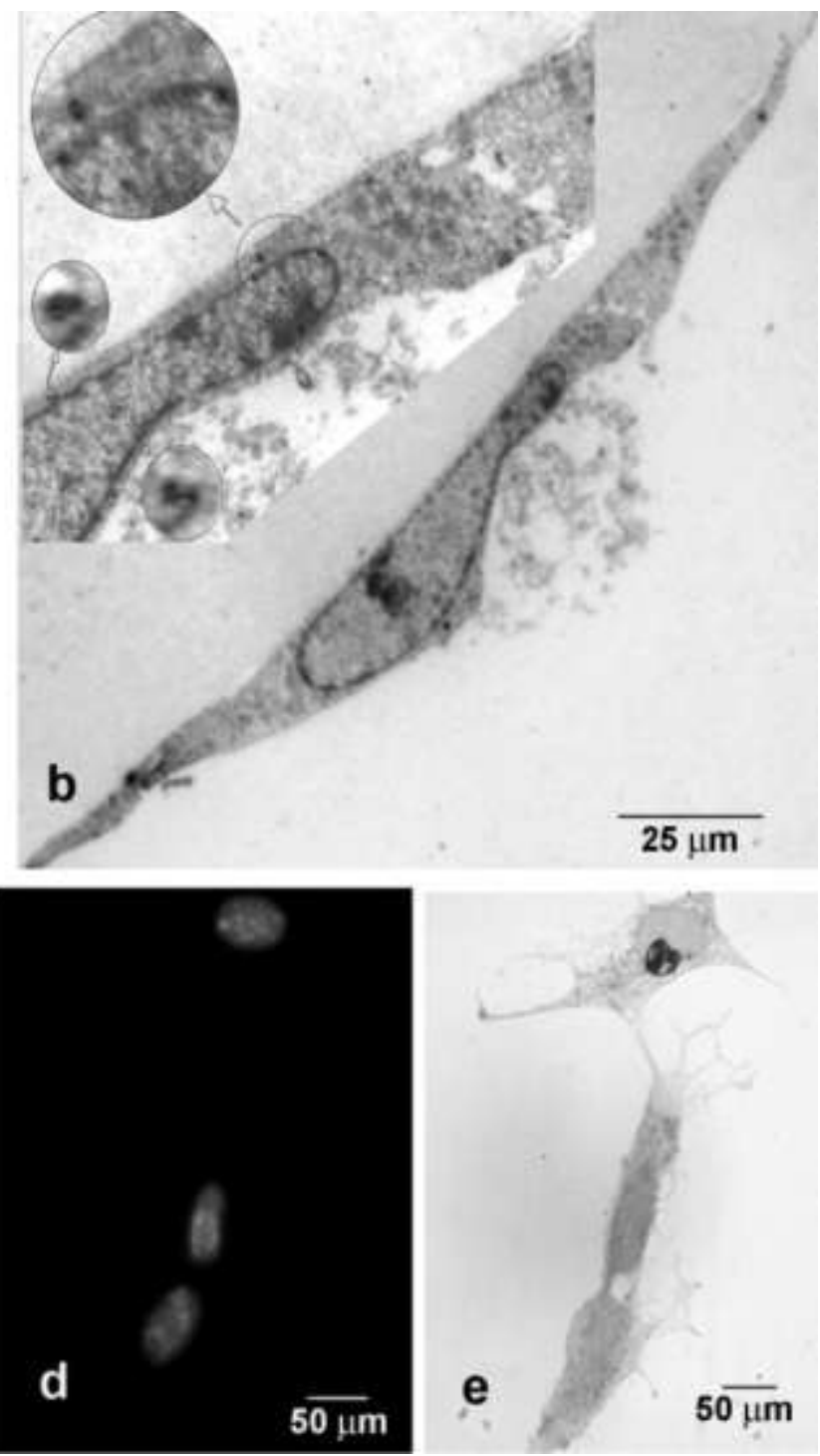

Figure 1. HUVEC infection by HHV-8: a Time course of HHV-8 replication. HHV-8 DNA accumulation peaked after 36-48h, decreased slowly till day 10 becoming undetectable at day 17, differently from UV inactivated HHV-8 mock, that decreased early. The grey area represents the threshold of sensitivity of the calibrated Real time PCR assay used ( $<10$ genome equivalents $/ \mathrm{mL}$ ). b Transmission electron microscopy of an infected endothelial cell grown on a polycarbonate filter and harvested at 36 hrs after HHV-8 infection. The different insets evidence viral-like particles distribution and cellular alterations (original magnifications: x 2500, x 5000, 25000). c, d, e confocal micrographs of infected cells at t1 showing viral IL-6 cytoplasmic expression (c; original magnification: $x$ 600), DAPI (d) and LANA-1 staining (e) on a small group of morphologically altered cells (original magnifications: $x 400$ ). 


\section{CTRL}
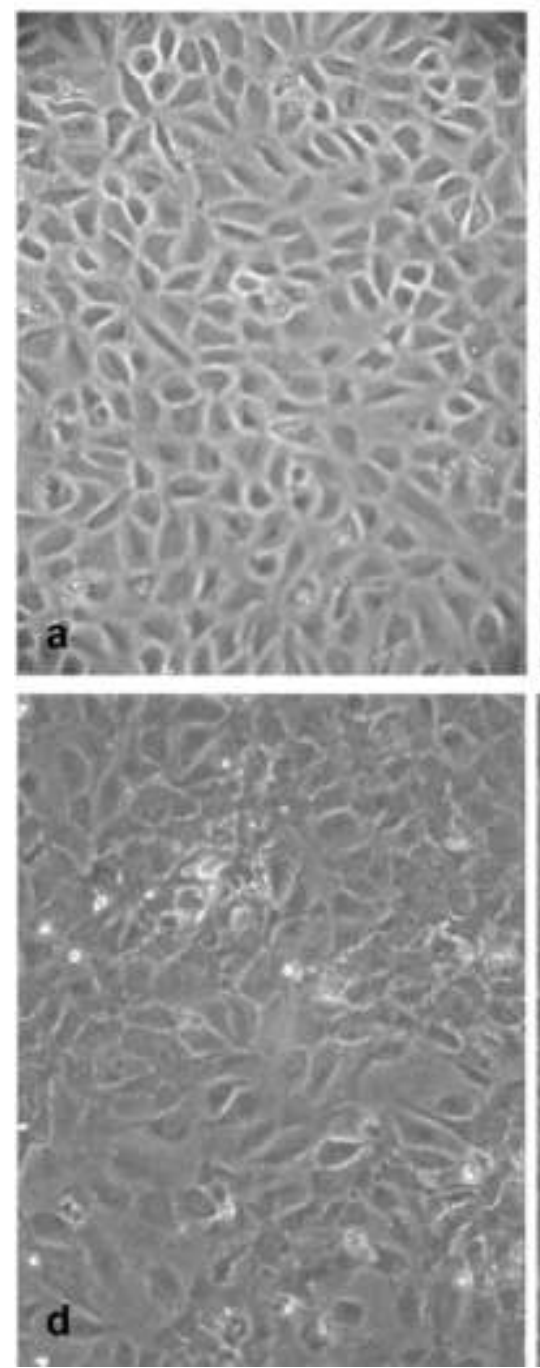

Mock
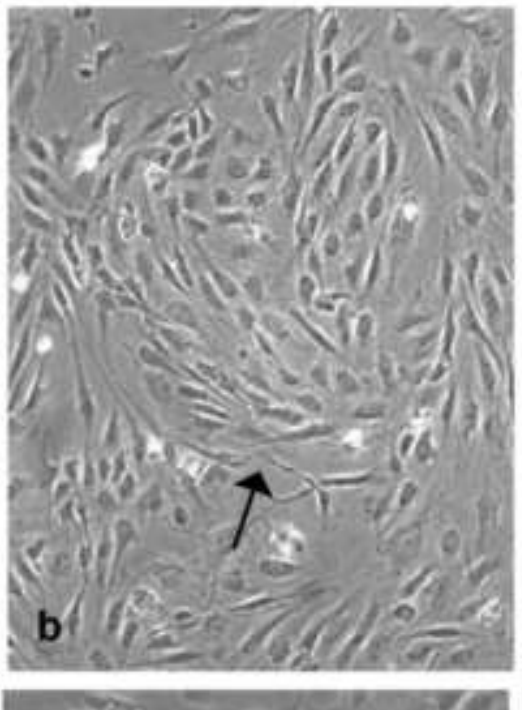

Infected
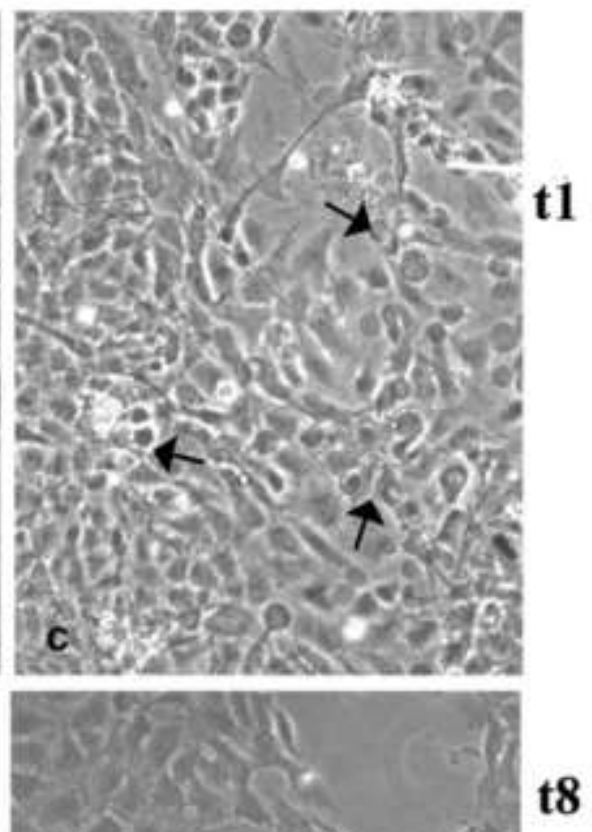

t8

Figure 2. HUVEC morphology upon infection by HHV-8. Phase contrast microscopy of cells untreated (a, d) treated with UV-inactivated HHV-8 (b, e) or with infectious HHV-8 supernatant (c, f) at $t 1$ (first row), and at t8 (second row). Arrows indicate in b, a spiralized area, in $c$ rounded cells and in $f$ few cells in the upper layer of cordon-like structure (original magnifications: $x 200$ ).

\section{Morphological changes occuring in HHV-8 infect- ed HUVEC}

The morphological changes accompanying HHV8 infection of HUVEC were followed daily with contrast-phase microscope and compared with the morphology of both control monolayer (untreated) and monolayer exposed to UV-inactivated virus. At t1, control cultures displayed the classical features of normal HUVEC monolayers: strictly adjacent clear polygonal cells, well spread and homogenously distributed (Figure 2a). By contrast, cells treated with either live or UV-inactivated virus showed evident morphological changes. Cells treated with UV-inactivated virus exhibited a smaller and elongated shape (spindle-like) and grew forming a plane spiral already during the first 24 hours post-treatment (Figure 2b, arrow). In comparison, infected cultures grew with a more disorganized pattern (Figure 2c): the cells were irregular in shape and size, with elements characterized by elongated and thin protrusions in close contact with rounded cells (Figure 2c, arrows). After 1 week the morphology of cultures treated with UV-inactivated virus partially reverted to a normal appearance, mainly showing polygonal cells not in close contact (Figure 2e); subsequently, there was a complete monolayer rescue (not shown). By contrast, the loss of culture organization in infected specimens worsened with time (Figure 2d), accompanied by a significant cell mortality $(\geq 30-40 \%$ ) between $\mathrm{t} 3$ and $\mathrm{t} 5$ (not 


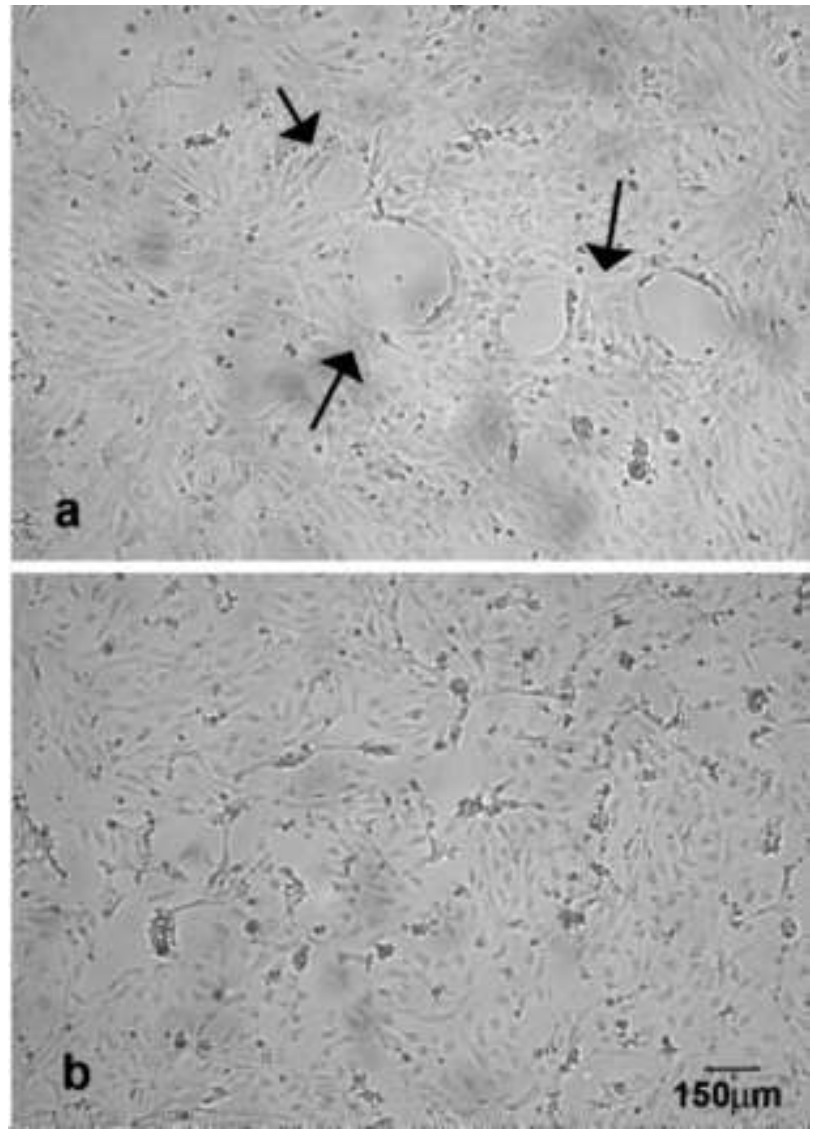

Figure 3. HUVEC morphology at t20 after infection by HHV-8. The presence of $2 \mathrm{D}$ structures that resemble capillaries (arrows) (a) or of granulous dying cells (b) (original magnification: $\mathbf{x 1 0 0 )}$ is shown.

shown). After 1 week of HHV-8 infection, HUVEC appeared dramatically altered in both shape and growth rate. The cells lost the contact inhibition and started to grow in multiple layers: the upper layer was mainly composed by cordons of (Figure 2f), whereas the lower layer mantained a more regular organization and a more conserved cellular shape. Infected cells survived in culture for several days with a reduced growth rate, but without showing additional mortality. At t20, surviving cells alternated areas of the monolayers forming two-dimensional structures resembling capillary like structures (Figure $3 a$, arrows) with areas of disorganization containing high numbers of dying cells (Figure $3 \mathrm{~b}$ ) followed few days later by a massive cell-death and loss of the infected cultures, both occurring also in the uninfected controls.

Thus, in our model, UV-inactivated HHV-8 supernatant induced only transient changes in HUVEC cultures, whereas infectious virus induced irreversible alterations.

\section{Phenotypic changes in HHV-8-infected HUVEC}

To assess whether in our system the morphological alterations were accompanied by modifications of the endothelial cell phenotype, we investigated the expression and localisation of several endothelial cell markers, in combination with the cytoskeletal arrangement and cellular proliferation status by confocal microscopy. Twenty-four hours after H HV8 infection, the normal distribution of CD31 /PECAM1, which decorates the interendothelial junctions in uninfected cultures (Figure 4a), was altered, both with regard to its whole culture distribution and its single-cell expression level. Areas brigthly expressing CD31/PECAM1, corresponding to the cordon-like structures, were observed next to areas of low CD31/PECAMI expression (Figure $4 b)$. Notably, the free margins of the cells at the periphery of the cordons showed an unusually high expression of CD31/PECAM, indicating an activated endothelial cell phenotype. The altered distribution of CD31/PECAM1, consistent with the profound disorganization of the endothelial cultures, was accompanied by a significant modification in
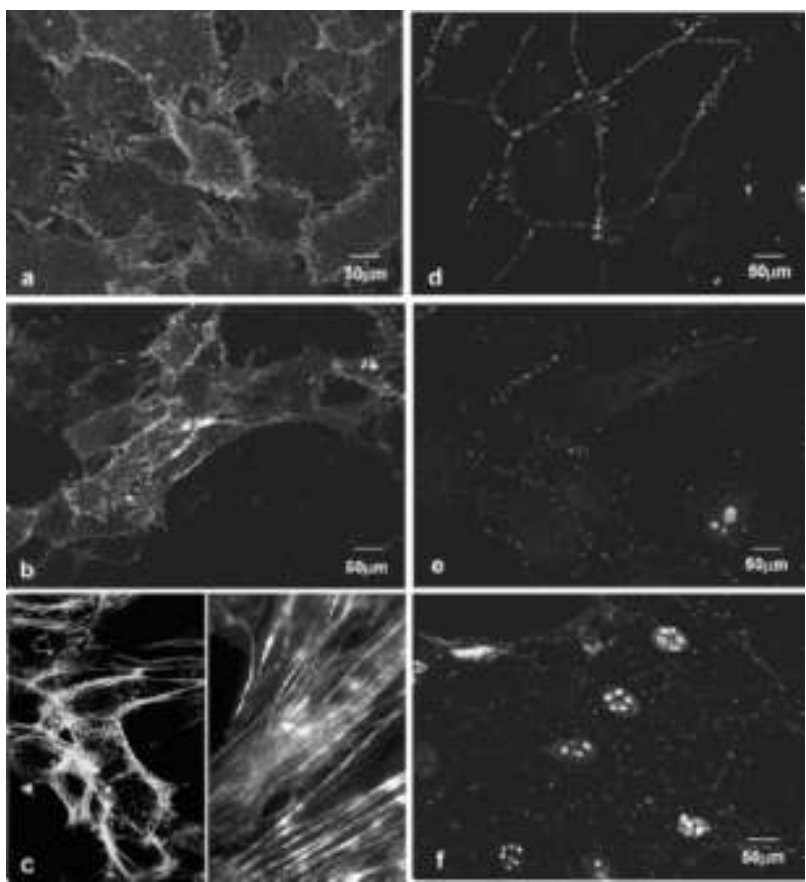

Figure 4. HUVEC phenotype upon infection by HHV-8. Confocal microscopy of CD31/PECAM (a) , F-actin (c, left panel), VE-cad and Ki67 (d) in uninfected HUVEC. CD31/PECAM (b); F-actin (c, right panel), VE-cad and $\operatorname{Ki67}(e, f)$ at to $(e)$ and t7 $(b, c)$ in infected cells. An increase of CD31 signal is evident in $b$, the loss of VE-cadherin expression accompanied by the increase in the number of Ki67 positive cells is shown in $\mathrm{f}$, and the presence of stress fibers displayed by cordon cells is evident in c (right panel) compared with typical peripheral bundles of quiescent HUVEC (left panel)(original magnification: x 600). 


\section{uPAR expression}

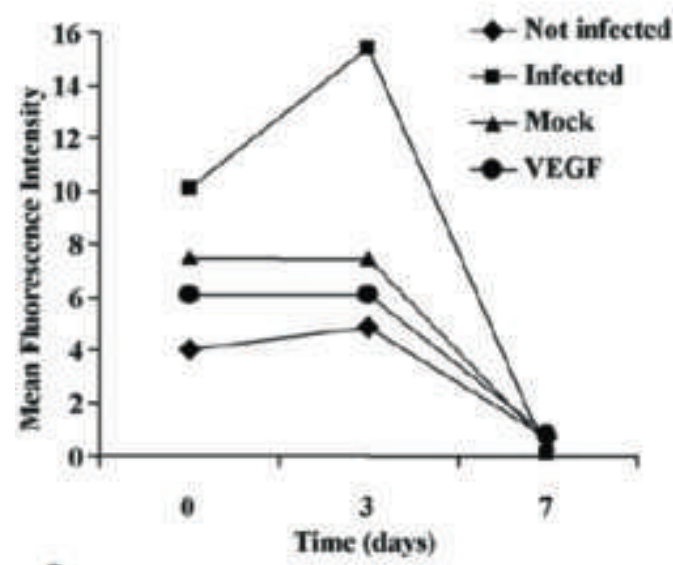

a
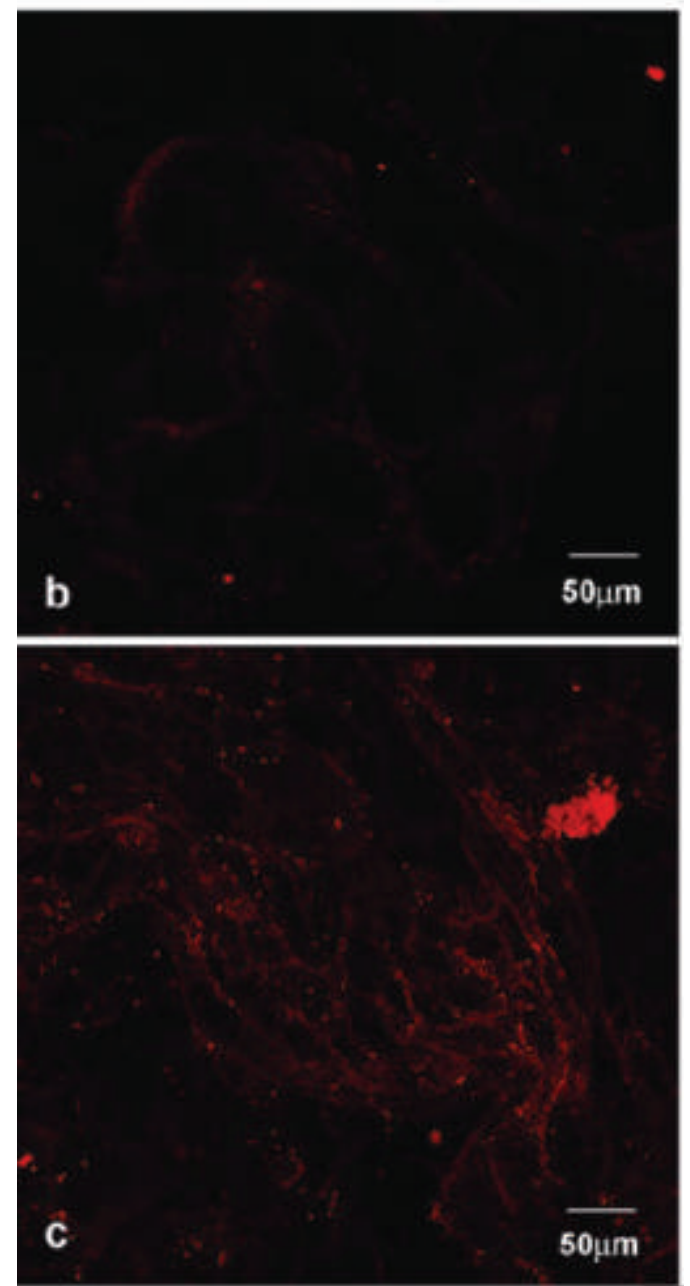

Figure 5. Angiogenic markers expression in cordon-like structures at day 7 upon HHV-8 infection. a: Time course analysis of uPAR expression, in cells uninfected (black diamond), VEGFtreated (black circle), and treated with UV-inactivated (black triangle) or infectious HHV-8 BCBL-1 supernatant (black square). Noteworthy, uPAR expression was already significantly up-regulated after $0 / \mathrm{N}$ infection (t0). b, c: Analysis of CD13/APN expression. Expression of the CD13/APN molecule is restricted to HUVEC cells in the upper layer of the cordon-like structures (original magnification: x 600). the cytoskeletal arrangement. Indeed, at $\mathrm{t} 7$, the infected cells corresponding to the lower layer displayed quiescent F-actin peripheral bundles (Figure $4 c$ left), while those in the upper layer displayed stress fibres, again indicating endothelial cell activation (Figure $4 c$ right).

To better define the magnitude of the endothelial modifications, we investigated the distribution of another specific endothelial cell-molecule, VE-cad, which is involved in the formation and maintenance of the interendothelial junctions. In control HUVEC, VE-cad decorates the intercellular profile, lining the finger-like contacts typical of a confluent monolayer (Figure 4d). Upon infection, VE-cad signal progressively decreased and adopted a peculiar, dot-like pattern, mainly occurring in cordon structures (Figure 4e). Consistently, the proliferation rate of the cells belonging to the cordons increased, as showed by Ki67 co-staining (Figure $4 d-f$ ).

In conclusion, infected HUVEC showed signs of functional activation, such as proliferation and stress-fibers generation, together with the loss of the specific lineage markers, CD31/PECAMI and VE-cad.

\section{Infected HUVEC show changes reminiscent of an angiogenic transformation}

To better characterize the nature of the changes induced by $\mathrm{HHV}$-8, we investigated whether infected HUVEC had undergone an angiogenic switch. To this end, we examined the expression of UPAR, which is involved in the process of extracellular matrix proteins degradation and cell-migration, both processes preliminary to the formation of new vessels (Kim et al., 2003; Trisciuoglio et al., 2004). UPAR expression, as demonstrated by FACS analysis, increased in the early stages of infection (from t0 to t3) (Figure 5a), indicating a propensity of the cells to move, but was lost after one week of HHV8 infection. All the control cultures (untreated, treated with UV-inactivated virus or treated with VEGF), displayed a lower UPAR expression level and a negligible increase over time (Figure $5 \mathrm{a}$ ). Analogously, CD13/APN, a membrane-bound metalloprotease that is expressed on the endothelial cells of angiogenic but not normal vessels, was expressed by HUVEC forming the upper layer cordon-like structures in infected cultures (Figure 5c), but not by cells forming the lower layer (Figure $5 b$ ). Interestingly, DAPI staining showed that the cordon structures were formed by 30-50 strictly packaged 

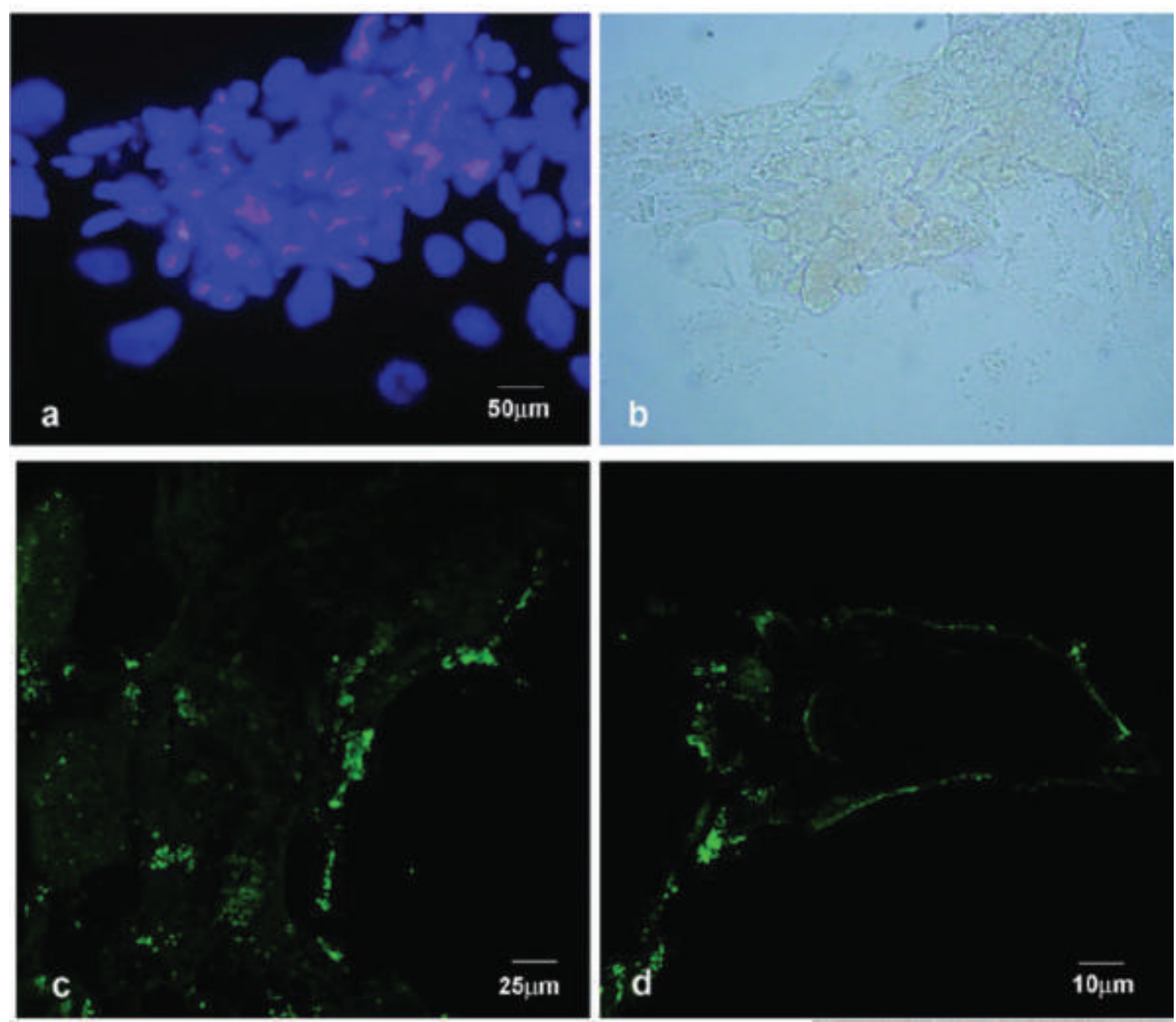

Figure 6. HHV-8 antigen expression in cordon-like structures. Microscopy of HUVEC cordon structures stained with DAPI (a) and antiLANA-1 (IHC) (b) or with anti-v-IL6 (confocal microscopy) (e, d) at $t 7$ after infection. The cordons are formed by a hight number of cells weakly expressing LANA-1, whereas a minority of cells located at the periphery expressed v-IL6; a higher magnification of a v-IL6 positive cell is shown in d (original magnification: x 400 in a, b; x 600 in e; x 1200 in d).

cells disposed irregularly in 2-3 layers, with normal nuclei (Figure 6a). Notably, only within the upper layer most nuclei weakly expressed the LANA-1 antigen (Figure 6b), while fewer cell, localized in the periphery, expressed v-IL6 (Figure 6c, d), indicating a direct involvement of $\mathrm{HHV}-8$ in the neoangiogenetic process.

\section{Discussion}

Using complementary experimental approaches, we confirmed that primary human endothelial cells can be productively infected by $\mathrm{HHV}-8$, a virus linked to the etiology of peculiar tumors characterized by marked neoangiogenesis. More importantly, we provided evidence that the presence of actively replicating HHV-8 drives consistent morphologic and phenotypic changes in cultures of primary human endothelial cells, which resemble the first steps of angiogenic transformation.

Similarly to other in vitro models (Ciufo et al., 2001; Gao et al., 2003), our experimental system is characterized by the coexistence of cells showing signs of both the latent and lytic phases of the HHV-8 life-cycle, as well as by an important cell depletion after 3-5 days of culture and by the appearance of spindle-like cells early after infection. However, we have also observed an angiogenic transformation of endothelial cell cultures never described before in $\mathrm{HHV}$-8-infected model systems, 
with the formation of foci of irregularly-shaped cells, which lost contact inhibition and grew forming multilayers in cordon-like structures consisting of 30 to 50 cells. These structures were directly linked to HHV-8 replication, as suggested by the expression of VIL-6 and by the persistent HHV-8 DNA release in the culture supernatant. In fact, the spindle-like morphology cannot be considered as a bona fide H HV-8-driven phenomenon since, in our hands, it occurred also in cells treated with UVinactivated HHV-8, and it has been obtained pulsing normal endothelial cells with inflammatory cytokines (Ensoli et al., 1998, 2001; Samaniego et al., 1998). These cordon-like formations were characterized by a number of morphologic and phenotypic changes, such as redistribution of CD31 IPECAMI molecule, loss of VE-cad expression, and cytoskeletal re-organization accompanied by de novo expression of the CD13/APN molecule.

PECAM-1, a member of the Ig superfamily, is expressed on endothelial cells at the level of the intercellular junctions (Newman, 1997; Sun et al., 2000; Feng et al., 2004; Dejana , 2004), contributing to create and maintain the endothelial barrier function (Ferrero et al.,1995, 1996; Zocchi et al., 1996; Nakada et al., 2000). Moreover, PECAMI is able to coordinate the assembly of Factin filaments occurring in cell-reshape and migration (Ohmori et al., 2001; Poggi et al., 1996; Matsumara et al., 1997). Finally, PECAMI plays a role in tumor-associated angiogenesis in various animal models (DeLisser et al., 1997) and is involved in the ability of endothelial cells to form three dimensional networks in Matrigel (Sheibani et al., 1997). Altogether, these features suggest that PECAMI actively partecipates to the angiogenetic machinery. Therefore, the altered distribution and partial loss of PECAMI expression, observed in HHV-8 infected HUVEC, together with the regular arrangement along the cellular major axes of the cytoskeletal fibers occurring in cordonlike structures are suggestive of an ongoing angiogenic switch. This hypotesis is strengthened by the presence of reduced level of VE-cad on the same type of cells and its unique pattern of surface distribution. On endothelial cells, VE-cad (Lampugnani et al., 2003) is specifically located at the adherens junctions forming zipper-like structures along the intercellular contacts (Figure $4 d$ ). This protein is linked through its cytoplasmic tail to p120, $\beta$-catenin, and $\gamma$-catenin (Gottardi and
Gumbiner, 2001), which, in turn, mediate the anchorage of VE-cadherin to the actin cytoskeleton. VE-cadherin expression and clustering at intercellular junctions blocks the proliferative response of the cells to VEGF (D'Amore, 1992; Vinals and Pouyssegur, 1999), suggesting that VE-cadherin may transduce growth-inhibitory signals (Vinals and Pouyssegur, 1999; Fagotto andd Gumbiner, 1996). Indeed, contact inhibition of VEGF-induced proliferation requires the integrity of the vascular endothelial cadherin, $\beta$-catenin, and the phosphatase DEP-1/CD148 network (Caveda et al., 1996). Noteworthy, in our experimental setting, the cells grouped in the cordon-like structures lost the contact inhibition, displaying a profound remodelling of VE-cad in concomitance with the acquisition of a high rate of proliferation. Indeed, the perturbation of the lateral localization of VE-cad, similar to the one found after $\mathrm{Ca}^{++}$chelation or sialidase treatment, substantiates the tendency of the cells to migrate.

On the whole, the profound changes observed raise the question of whether HHV-8 infected HUVEC initiate an angiogenic process. Angiogenesis is a highly organized process in which growth factors like basic fibroblast growth factor-2 $(\beta-F G F-2)$ and vascular endothelial growth factorA (VEGF-A) stimulate endothelial cells to secrete matrix metalloproteinases, migrate, proliferate, and finally differentiate to form a capillary (Marchio et al., 1999; Bussolino et al., 1997). In this context, the expression of the urokinase-type plasminogen activator receptor (UPAR) and of the CD13/APN plays a crucial role in regulating the angiogenic program. In fact, UPAR localizes UPA at the cell surface making cell-associated proteolysis focally oriented. Similarly, the CD13/APN metalloprotease is expressed de novo only on angiogenic but not on normal vessels (Bhagwat et al., 2003). Moreover, treatment with CDI3/APN functional inhibitors arrested retinal neovascularization, chorionallantoic membrane angiogenesis and xenograft tumor growth in animal models (Bhagwat et al., 2001). The marked expression of CD13/APN on cordonforming cells, as well as the timely regulated induction of UPAR occuring only in HHV-8 infected cultures, provide a strong argument in favor of a H HV8 driven angiogenic switch.

Following the exposure to HHV-8, we have not achieved a frank neoplastic transformation of HUVEC cells since we could not establish 
monomorphic continuous cell lines. On the other hand, several lines of evidence suggest that, in vivo, $\mathrm{KS}$ in its early stage is not a true sarcoma but rather a hyperplastic reactive process mediated in part by inflammatory cytokines and angiogenic factors triggered by HHV-8 infection. Indeed, KS lesions can simultaneously appear with a symmetrical localisation in the absence of metastases; the lesions are characterized by a polyclonal profile of both androgen receptor gene methylation and HHV-8 genomic terminal repeats (TR) (Judde et al., 2000; Gill et al., 1998) in the absence of signs of aneuploidy (Gill et al., 1998). In addition, KS can regress spontaneously or, in AIDS patients, following the administration of highly active antiretroviral therapy (HAART) (Janier et al., 1985; Lebbè et al., 1998). Therefore, it is likely that the progression of KS into a real monomorphic sarco$\mathrm{ma}$, as sustained in advanced KS lesions by the findings of aneuploidy, microsatellite instability and clonality of Kaposi spindle cells (Bedi et al., 1995; Rabkin et al., 1995; Rabkin et al., 1997; Herndier et al., 1994), may require additional factors besides HHV-8, such as a permissive microenvironment, mainly consistent of cytokines, angiogenic and cellular growth factors, capable of promoting the expression of cellular oncogenes and of timely modulating virally encoded oncogenes and promoting factors. Nevertheless, this model offers a clear evidence for a direct HHV-8 involvement in the initiation and establishment of a series of early angiogenic changes that may be relevant to KS pathogenesis.

\section{Acknowledgments}

We wish to thanks Sara Piergiovanni for technical assistance in Real-time PCR, and Elena Dalcin and Marianna Gaman for technical support in TEM analysis.

\section{References}

Bedi GC, Westra WH, Farzadegan H, Pitha PM, Sidransky D. Microsatellite instability in primary neoplasms from HIV+ patients. Nat Med. 1995; 1:65-68.

Bhagwat SV, Lahdenranta J, Giordano R, Arap W, Pasqualini R, Shapiro LH. CD13/APN is activated by angiogenic signals and is essential for capillary tube formation. Blood. 2001; 97(3): 652-9.

Bhagwat SV, Petrovic N, Okamoto $Y$, Shapiro LH. The angiogenic regulator CD13/APN is a transcriptional target of Ras signaling pathways in endothelial morphogenesis. Blood. 2003; 101(5): 1818-26.

Boshoff C, Schulz TF, Kennedy MM et al. Kaposi's sarcoma-associated herpesvirus infects endothelial and spindle cells. Nat Med. 1995; 1:1274-1278.
Boshoff C, Wiess RA. Kaposi's sarcoma-associated herpesvirus. Adv Cancer Res. 1998; 75:57-86.

Bosman C, Bisceglia M, Quirke P. Ultrastructural study of Kaposi's sarcoma. Pathologica. 1996; 88:8-17.

Broccolo F, Locatelli G, Sarmati L et al. Calibrated real-time PCR assay for quantitation of human herpesvirus 8 DNA in biological fluid. J Clin Microbiol. 2002; 40:4652-58.

Bussolino F, Mantovani A, Persico G. Molecular mechanisms of blood vessel formation. Trends Biochem Sci. 1997; 22:251-56.

Caveda L, Martin-Padura I, Navarro P et al. Inhibition of cultured cell growth by vascular endothelial cadherin (cadherin-5/VE-cadherin). J Clin Invest.1996; 98:886-93.

Cesarman E, Chang Y, Moore PS, Said JW Knowles DM. Kaposi's sarcoma-associated herpesvirus-like DNA sequences in AIDS-related body-cavity based lymphomas. N Engl J Med. 1995; 332:11861191.

Chang $Y$, Cesarman E, Pessin MS et al. Identification of herpesviruslike DNA sequences in AIDS-associated Kaposi's sarcoma. Science. 1994; 266:1865-1869.

Ciufo DM, Cannon JS, Poole LJ et al. Spindle cell convertion by Kaposi's sarcoma-associated herpesvirus: formation of colonies and plaques with mixed lytic and latent gene expression in infected primary dermal microvascular endothelial cell cultures. J Virol. 2001; 75:5614-5626.

D'Amore PA. Mechanisms of endothelial growth control. Am J Respir Cell Mol Biol. 1992; 6:1-8.

Dejana E. Endothelial cell-cell junctions: happy together. Nat Rev Mol Cell Biol. 2004; 5:261-70.

DeLisser HM, Christofidou-Solomidou M, Strieter RM et al. Involvment of endothelial PECAM-1/(CD31) in angiogenesis. Am J Pathol. 1997; 151:671-77.

Dourmishev LA, Dourmishev AL, Palmeri D, Schwartz RA, Lukac DM. Molecular genetics of Kaposi's sarcoma-associated herpesvirus (human herpesvirus-8) epidemiology and pathogenesis. Microbial Mol Biol Rev. 2003; 67:175-212.

Dupin N, Fisher C, Kellam P et al. Distribution of human herpervirus8 latently infected cells in Kaposi's sarcoma, multicentric Castleman's disease an primary effusion lymphoma. Proc Natl Acad Sci USA. 1999; 96:4546-4551.

Ensoli B, Nakamura S, Salahuddin SZ RC. AIDS-Kaposi's sarcomaderived cells express cytokine with autocrine and paracrine growth effects. Science 1989, 242: 223-226.

Ensoli B, Sgadari C, Barillari G, Sirianni MC, Strurzl M, Monini P: Biology of Kaposi's sarcoma. Eur J Cancer. 2001 ; 37:1251-69.

Fagotto F, Gumbiner BM. Cell contact-dependent signaling. Dev Biol. 1996; 180:445-54.

Feng D, Nagy JA, Pyne K, Dvorak HF, Dvorak AM. Ultrastructural localization of platelet endothelial cell adhesion molecule (PECAM1, CD31) in vascular endothelium. J Histochem Cytochem. 2004; 52:87-101.

Ferrero E, Bondanza A, Leone BE, Manici S, Poggi A, Zocchi MR. CD14+ CD34+ peripheral blood mononuclear cells migrate across endothelium and give rise to immunostimulatory dendritic cells. J Immunol. 1998; 160:2675-83.

Ferrero $E$, Ferrero ME, Pardi $R$, Zocchi MR. The platelet endothelial cell adhesion molecule-1 (PECAM1) contributes to endothelial barrier function. FEBS letters. 1995; 374:323-326.

Ferrero E, Villa A, Ferrero ME et al. Tumor Necrosis Factor $\alpha$-induced vascular leakage involves PECAMI phosphorylation. Cancer Res. 1996; 56:3211-3215.

Fiorelli V, Gendelman R, Samaniego F, Markham PD, Ensoli B. Cytokines from activated T cells induce normal endothelial cells to acquire the phenotypic and functional features of AIDS-Kaposi's sarcoma spindle cells. J Clin Invest. 1995; 95:1723-34.

Fiorelli V, Gendelman R, Sirianni MC et al. gamma-Interferon produced by CD8+-T cells infiltrating Kaposi's sarcoma induces spindle cells with angiogenic phenotype and synergy with HIV-1 Tat protein: an immune response to HHV-8 infection? Blood. 1998; 91:956967.

Flore 0, Rafii S, Ely S, O'Leary JJ, Hyjek EM, Cesarman E. Transformation of primary human endothelial cells by Kaposi's sarcoma-associated herpesvirus. Nature. 1998; 394:588-592,

Gao SJ, Deng JH, Zhou FC et al. Productive lytic replication of a recombinant Kaposi's sarcoma-associated herpesvirus in efficient 
primary infection of primary human endothelial cells. J Virol. 2003; 77: 9738-49.

Gill PS, Tsai YC, Rao AP. Evidence for multiclonaliy in multicentric Kaposi's sarcoma. Proc Natl Acad Sci USA. 1998; 15:8257-61.

Gottardi CJ, Gumbiner BM. Adhesion signaling: how beta-catenin interacts with its partners. Curr Biol. 2001; 11:R792-94.

Herndier BG, Werner A, Arnstein P et al. Chracterization of a human Kaposi's sarcoma cell line that induces angiogenic tumors in animal. AIDS. 1994; 8:575-81.

Janier M, Vignon MD, Cottenot F. Spontaneously healing Kaposi's sarcoma in AIDS. N engl J Med. 1985; 312:1638-39.

Judde JG, Lacoste $V$, Briere J. Monoclonality or oligoclonality of human herpesvirus 8 terminal repeat sequences in Kaposi's sarcoma and other diseases. J Natl Cancer Inst. 2000; 92:729-36.

Kim KS, Hong YK, Lee Y et al. Differential inhibition of endothelial cell proliferation and migration by urokinase subdomains: amino-terminal fragment and kringle domain. Exp Mol MED. 2003; 35:578-85.

Lampugnani $M G$, Zanetti $A$, Corada $M$ et al. Contact inhibition of VEGF-induced proliferation requires vascular endothelial cadherin beta-catenin, and the phosphatase DEP-1/CD148. J Cell Biol. 2003; 161:793-804.

Lebbè $C$, Blum L, Pellet $C$ et al. Clinical and biological impact of antiretroviral therapy with protease inhibitors on HIV-related Kaposi's sarcoma. AIDS. 1998; 12:F45-49.

Locatelli G, Santoro F, Veglia F, Gobbi A, Lusso P, Malnati MS. Realtime quantitative PCR for human herpesvirus 6 DNA. J Clin Microbiol. 2000; 38:4042-48.

Lusso P, Malnati M, De Maria A, Balotta C, DeRocco SE, Markham $P D$, et al. Productive infection of CD4+ and CD8+ mature human $T$ cell populations and clones by human herpesvirus 6 . Transcriptional down-regulation of CD3. J Immunol. 1991 Jul 15;147(2):685-91.

Malnati MS, Dagna L, Ponzoni M, Lusso P: Human herpesvirus 8 (HHV8/KSHV) and hematologic malignancies. Rev Clin Exp Hematol. 2003; 7:375-405.

Marchio S, Primo L, Pagano M. Vascular endothelial growth factor-C stimulates the migration and proliferation of Kaposi's sarcoma cells. J Biol Chem. 1999; 274:27617-22.

Matsumara T, Wolff K, Petzelbauer P: Endothelial cell tube formation depends on cadherin 5 and CD31 interactions with filamentous actin. J Immunol. 1997 ; 158:3408-16.

Mercader M, Taddeo B, Panella JR, Chandran B, Nickoloff BJ, Foreman KE. Induction of H HV-8 lytic cycle replication by inflammatory cytokines produced by HIV-1-infected T cells. Am J Pathol 2000. 156;1961-1971.

Michaux G, Cutler DF. How to roll an endothelial cigar: the biogenesis of Weibel-Palade bodies. Traffic. 2004; 5:69-78.

Nakada MD, Amin K, Christofidou-Solomidou M et al. Antibodies against the first Ig-like domain of human platelet endothelial cell adhesion molecule-1 (PECAM) that inhibit PECAM-1-dependent homophilicadhesion blockin vivo neutrophil recruitment. J Immunol. 2000; 164:452-62.

Neipel F, Fleckenstein B. The role of HHV-8 in Kaposi's sarcoma. Semin Cancer Biol. 1999; 9:151-164.

Newman PJ. The biology of PECAM-1. J Clin Invest. 1997; 99:3-8.

Nickoloff BJ, Griffiths CE. Factor XIII-expressing dermal dendrocytes in AIDS-associated cutaneous Kaposi's sarcomas. Science. 1989; 243:1736-1737.
Ohmori T, Yatomi Y, Wu Y, Osada M, Satoh K, Ozaki Y. Wheat germ agglutinin-induced platelet activation via platelet endothelial cell adhesion molecule-1: involvement of rapid phospholipase $\mathrm{C}$ gamma 2 activation by Src family kinases. Biochemistry. 2001; 40:129923001.

Poggi A, Panzeri MC, Moretta L, Zocchi MR. CD31-triggered rearrangementof the actin cytoskeleton in human natural killer cells. Eur J Immunol. $1996 ; 26: 817-24$.

Rabkin CS, Bedi G, Musaba E et al. AIDS-related Kaposi's sarcoma is a clonal neoplasm. Clin Cancer Res. 1995; 1:257-60.

Rabkin CS, Janz S, Lash A et al. Monoclonal origin of multicentric Kaposi's sarcoma lesions. N Engl J Med. 1997, 336:988-93.

Samaniego F, Markham PD, Gendelman R et al. Vascular endothelial growth factor and basic fibroblast factor present in Kaposi's sarcoma are induced by inflammatory cytokines and synergize to induce vascular permeability and KS lesion development. Am J Pathol. 1998; 152:1433-43.

Sheibani N, Newman PJ, Frazier WA. Thrombospondin-1, a natural inhibitor of angiogenesis, regulates platelet-endothelial cell adhesion molecule-1 expression and endothelial cell morphogenesis. Mol Biol Cell. 1997; 8:1329-41.

Soulier J, Grollet L, Oksenhendler E et al. Kaposi's sarcoma-associated herpesvirus-like DNA sequences in multicentric Castleman's disease. Blood. 1995; 86:1276-1280.

Staskus KA, Zhong W, Gebhard K et al. Kaposi's sarcoma- associated herpesvirus gene expression in endothelial (spindle) tumor cells. J Virol. 1997; 71:715-719.

Sturzl M, Blasig C, Schreier A et al. Expression of HHV-8 latencyassociated T0.7 RNA in spindle cells and endothelial cells of AIDSassociated, classical and African Kaposi's sarcoma. Int $\mathrm{J}$ Cancer. 1997; 72:68-71.

Sturzl M, Hohenadl C, Zietz C et al. Expression of K13/v-FLIP gene of human herpesvirus 8 and apoptosis in Kaposi's sarcoma spindle cells. J Natl Cancer Inst. 1999; 91:1725-1733.

Sturzl M, Wunderlich A, Ascherl G et al. Human herpesvirus-8 (HHV8) gene expression in Kaposi's sarcoma (KS) primary lesions: an in situ hybridization study. Leukemia. 1999; 13:S110-S112.

Sun J, Paddock C, Shubert $J$ et al. Contribution of the extracellular and cytoplasmic domains of platelet-endothelial cell adhesion molecule-1 (PECAM-1/CD31) in regulatimg cell-cell localization. J Cell Sci. 2000; 113:1459-69.

Trisciuoglio D, Iervolino A, candiloro A et al. bcl-2 induction of urokinase plasminogen activator receptor expression in human cancer cells through Spl activation: involvement of ERK1/ERK2 activity. J Biol Chem. 2004; 279:6737-45.

Uccini S, Ruco LP, Monardo F et al. Co-expression of endothelial cell and macrophage antigens in Kaposi's sarcoma cells. J Pathol. 1994; 173:23-31.

Uccini S, Sirianni MC, Vincenzi L et al. Kaposi's sarcoma (KS) cells express the macrophage associated antigen mannose receptor and develop in peripheral blood cultures od KS patients. Am J Pathol. 1997; 150:929-938.

Vinals F, Pouyssegur J. Confluence of vascular endothelial cells induces cell cycle exit by inhibiting p42/p44 mitogen activated protein kinase activity. Mol Cell Biol. 1999; 19:2763-72

Zocchi MR, Ferrero E, Leone BE et al. CD31/PECAM-1 driven chemokine-independent transmigration of human $T$ lymphocytes. Eur J Immunol. $1996 ; 26: 759-767$. 